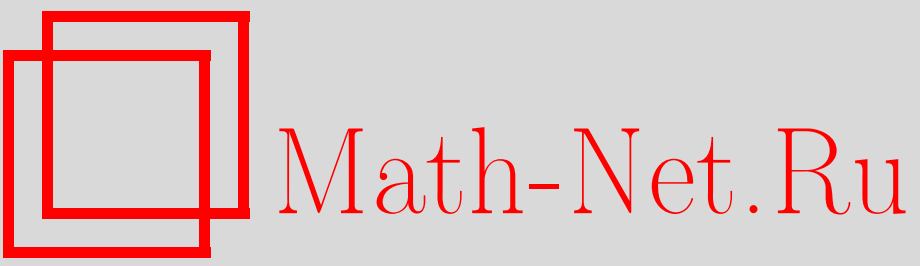

С. В. Талалов, Замечание о геометрическом описании релятивистской струны, TMФ, 2000, том 123, номер 1, 38-43

DOI: https://doi.org/10.4213/tmf583

Использование Общероссийского математического портала Math-Net.Ru подразумевает, что вы прочитали и согласны с пользовательским соглашением

http://www . mathnet.ru/rus/agreement

Параметры загрузки:

IP : 3.89 .197 .203

26 апреля 2023 г., 12:06:44 


\section{ЗАМЕЧАНИЕ О ГЕОМЕТРИЧЕСКОМ ОПИСАНИИ РЕЛЯТИВИСТСКОЙ СТРУНЫ}

Рассматривается теория классической трехмерной релятивистской струны в терминах квадратичных форм мирового листа. Показано, что существенным является учет не только первой, но и второй квадратичной формы. Получена система нелинейных эволюционных уравнений, конформно-инвариантным образом описывающая струнную динамику на поверхности первичных связей. Результаты обобщаются на $4 D$-случай.

Пусть $\mathbf{X}=X_{\mu}\left(\xi^{0}, \xi^{1}\right) \mathbf{b}^{\mu}$ - радиус-вектор мирового листа бесконечной ${ }^{1)}$ релятивистской струны в плоском псевдоевклидовом пространстве $E_{1,2}$. Хорошо известно [1], что параметризация $\xi^{0}, \xi^{1}$ всегда может быть выбрана конформно-плоской, т.е. так, что

$$
\left(\frac{\partial X}{\partial \xi^{0}}\right)^{2}>0
$$

почти всюду, поля $X_{\mu}\left(\xi^{0}, \xi^{1}\right)$ удовлетворяют свободным уравнениям $\partial_{+} \partial_{-} X_{\mu}=0$, а также связям $\left(\partial_{ \pm} X_{\mu}\right)^{2}=0$. Символами $\partial_{ \pm}$обозначены производные по “конусным" параметрам $\xi_{ \pm}=\xi^{1} \pm \xi^{0}$. Уравнения движения и связи инвариантны относительно преобразований конформной группы двумерного пространства-времени $\left(\xi^{0}, \xi^{1}\right)$ :

$$
\xi_{ \pm} \longrightarrow \widetilde{\xi}_{ \pm}=A_{ \pm}^{\prime}\left(\xi_{ \pm}\right), \quad A_{ \pm}^{\prime}>0
$$

В работе [2] показано, что уравнения Гаусса и Петерсона-Кодащци для мирового листа рассматриваемой струны могут быть сведены к единственному уравнению Лиувилля (УЛ) $\partial_{+} \partial_{-} \varphi=e^{\varphi}$. При этом был рассмотрен класс струнных конфигураций такой, что каждая орбита группы (1) содержит мировой лист, имеющий всюду постоянную вторую квадратичную форму $\mathbf{I I}=\rho_{i j} d \xi^{i} d \xi^{j}$. Между тем такой класс не является максимально общим. Действительно, предположим, что существуют сегменты $\left[a_{ \pm}, b_{ \pm}\right]$такие, что $\rho_{i j} \equiv 0$ при $\xi_{+} \in\left[a_{+}, b_{+}\right], \xi_{-} \in\left[a_{-}, b_{-}\right]$, причем в других точках $\rho_{i j} \neq 0$. В этом случае, вообще говоря, никаким преобразованием (1) форму II нельзя сделать постоянной всюду. Заметим, что соответствующий вывод УЛ, предложенный в статье [3], также

\footnotetext{
1) Рассмотрение для открытой и периодической струны аналогично.

${ }^{*}$ Филиал Самарского государственного педагогического института им. В. В. Куйбышева, Тольятти, Самарская обл., Россия
} 
не работает для указанного примера. В предлагаемой работе исследуется максимально обший случай, который, как будет показано, имеет сушественные отличия.

Рассматриваемая струнная теория является масштабно-инвариантной в пространстве $E_{1,2}$. Поэтому далее мы будем использовать в основном проективные переменные $x_{\mu}=X_{\mu} / p$, где $p$ - некоторая константа, имеюшая размерность длины $(p>0)$. Сделаем необходимые вспомогательные построения. Для этого используем тот факт, что всегда существуют светоподобные векторы $\mathbf{n}_{ \pm}\left(\xi^{0}, \xi^{1}\right)$, обладаюшие свойствами

$$
\begin{aligned}
n_{ \pm}^{\mu} \partial_{ \pm} x_{\mu} & = \pm \frac{1}{2}, \\
\partial_{ \pm} n_{\mp}^{\mu} & =0 .
\end{aligned}
$$

Произвол в их выборе исчерпывается преобразованиями из групп $G_{ \pm} \subset S O(1,2)$, которые являются стационарными для векторов $\partial_{+} x_{\mu}$ и $\partial_{-} x_{\mu}$, соответственно. Явная форма таких преобразований такова:

$$
n_{ \pm}^{\mu} \longrightarrow \widetilde{n}_{ \pm}^{\mu}= \pm g_{ \pm}^{2} \partial_{ \pm} x^{\mu}+n_{ \pm}^{\mu}-g_{ \pm} z_{ \pm}^{\mu}
$$

где $z^{\mu}=\varepsilon^{\mu \nu \rho} n_{\nu} \partial x_{\rho}$, а $g_{ \pm}=g_{ \pm}\left(\xi_{ \pm}\right)-$произвольные дифференцируемые функции. Указанный произвол мы обсудим позже, а сейчас воспользуемся лишь фактом сушествования $\mathbf{n}_{ \pm}\left(\xi_{ \pm}\right)$.

Выберем для определенности в пространстве $E_{1,2}$ мнимое представление матрищ Дирака: $\Gamma^{0}=\sigma_{2}, \Gamma^{1}=i \sigma_{1}$ и $\Gamma^{2}=i \sigma_{3}$. Кроме этого, мы используем представление координат (проективных) векторов $\mathbf{x}$ в виде бесшпуровых матрищ $2 \times 2$ :

$$
\widehat{x}=-i x_{\mu} \Gamma^{\mu}=\left(\begin{array}{cc}
x_{2} & x_{1}-x_{0} \\
x_{1}+x_{0} & -x_{2}
\end{array}\right) .
$$

Для соответствия $\mathbf{x} \leftrightarrow \widehat{x}$ определим также вектор-матрицу $\widehat{\mathbf{E}}_{0}$, отвечаюшую выбранному трансляционно-инвариантному базису $\mathbf{b}^{\mu}: \widehat{\mathbf{E}}_{0}=-i \mathbf{b}_{\mu} \Gamma^{\mu}$, так что $\mathbf{x}=\operatorname{Tr} \widehat{x} \widehat{\mathbf{E}}_{0}$.

В силу сделанных выше определений в каждой точке мирового листа струны обе тройки векторов

$$
\left( \pm \partial_{ \pm} \mathbf{x}+\mathbf{n}_{ \pm}, \partial_{ \pm} \mathbf{x} \mp \mathbf{n}_{ \pm}, \mathbf{z}_{ \pm}\right)
$$

являются ортонормироваными в $E_{1,2}$. Соответствующие им вектор-матрицы $\widehat{\mathbf{E}}_{ \pm}$имеют вид

$$
\widehat{\mathbf{E}}_{+}=\left(\begin{array}{cc}
\mathbf{z}_{+} & -2 \mathbf{n}_{+} \\
2 \partial_{+} \mathbf{x} & -\mathbf{z}_{+}
\end{array}\right), \quad \widehat{\mathbf{E}}_{-}=\left(\begin{array}{cc}
\mathbf{z}_{-} & 2 \partial_{-} \mathbf{x} \\
2 \mathbf{n}_{-} & -\mathbf{z}_{-}
\end{array}\right) .
$$

Не ограничивая обшности, преобразования $\widehat{\mathbf{E}}_{0} \rightarrow \widehat{\mathbf{E}}_{ \pm}$мы можем считать собственными, заменив, если это не так, $\mathbf{z} \rightarrow-\mathbf{z}$. Поэтому в силу локального изоморфизма $S O(1,2) \approx S L(2, R)$ имеем

$$
\widehat{\mathbf{E}}_{ \pm}=T_{ \pm} \widehat{\mathbf{E}}_{0} T_{ \pm}^{-1}
$$

при некоторых $T_{ \pm}\left(\xi_{ \pm}\right) \in S L(2, R)$, определенных, вообше говоря, с точностью до знака. Рассмотрим матрицу

$$
K\left(\xi^{0}, \xi^{1}\right) \equiv T_{-}\left(\xi_{-}\right) T_{+}^{-1}\left(\xi_{+}\right) .
$$


Ее геометрический смысл - преобразование пространства $E_{1,2}$, переводящее базис $\widehat{\mathbf{E}}_{+}\left(\xi_{+}\right)$в базис $\widehat{\mathbf{E}}_{-}\left(\xi_{-}\right)$. Важен факт, что компоненты $K_{i j}$ являются релятивистскими инвариантами. Действительно, их трансляционная инвариантность очевидна, т.к. при построении мы исходили из афффинных векторов $\partial_{ \pm} X$. Что касается лоренц-преобразований пространства $E_{1,2}$, то в соответствии с (5) при таких преобразованиях $T_{ \pm} \rightarrow$ $T_{ \pm} B$, где $B \in S L(2, R)$, так что матрица $K$ инвариантна.

Введенное $S L(2, R)$-значное поле $K\left(\xi^{0}, \xi^{1}\right)$ удовлетворяет уравнению

$$
\partial_{-}\left(K^{-1} \partial_{+} K\right)=0
$$

которое представляет собой частный случай уравнения кирального поля модели Весса-Зумино-Новикова-Виттена $[4,5]$. Если поле принимает значения в компактной группе, действие модели для общего случая записывается в виде $S(K)+a W(K)$, где $S(K)$ - действие главного кирального поля, а многозначньй функционал $W(K)$ имеет однозначную вариацию (см., например, [6]). Заметим, что функционал $W$ не сушествует, вообше говоря, для некомпактных групп.

Чтобы построить действие для динамической системы (6), мы поступим следуюшим образом. Запишем разложение Гаусса для матришы $K\left(\xi^{0}, \xi^{1}\right)$ :

$$
K=\left(\begin{array}{cc}
1 & 0 \\
-\alpha_{-} & 1
\end{array}\right)\left(\begin{array}{cc}
\exp \left(-\frac{\varphi}{2}\right) & 0 \\
0 & \exp \left(\frac{\varphi}{2}\right)
\end{array}\right)\left(\begin{array}{cc}
1 & \alpha_{+} \\
0 & 1
\end{array}\right)
$$

которое, как известно, однозначно определяет вешественные числа $\varphi$ и $\alpha_{ \pm}$для всех $K \in$ $S L(2, R)$, за исключением тех матриц, у которых $K_{11}=0$. Последнее равенство может быть записано в виде

$$
\exp \left(-\frac{\varphi\left(\xi^{0}, \xi^{1}\right)}{2}\right)=0
$$

так что разложение (7) однозначно определяет сингулярные функции $\varphi=\varphi\left(\xi^{0}, \xi^{1}\right)$ и $\alpha_{ \pm}=\alpha_{ \pm}\left(\xi^{0}, \xi^{1}\right)$.

Исходя из (7) легко получить систему уравнений, которой должны удовлетворять поля $\varphi$ и $\alpha_{ \pm}$. При этом удобно явно выделить переменные $\rho_{ \pm}=\left(\partial_{ \pm} \alpha_{\mp}\right) e^{-\varphi}$. В итоге имеем

$$
\begin{aligned}
-\frac{1}{2} \partial_{+} \partial_{-} \varphi+\rho_{+} \rho_{-} e^{\varphi} & =0 \\
\partial_{ \pm} \rho_{\mp} & =0 \\
\partial_{ \pm} \alpha_{\mp}-\rho_{ \pm} e^{\varphi} & =0 .
\end{aligned}
$$

Данные уравнения суть уравнения Лагранжа-Эйлера для действия

$$
\mathcal{S}=\int d \xi^{0} d \xi^{1}\left[\frac{1}{4}\left(\partial_{+} \varphi\right)\left(\partial_{-} \varphi\right)+\rho_{+} \rho_{-} e^{\varphi}-\rho_{+} \partial_{-} \alpha_{+}-\rho_{-} \partial_{+} \alpha_{-}\right]
$$

Система (9)-(11) была впервые исследована в работе [7] для случая $\rho_{ \pm} \geqslant 0$, где она интерпретировалась как система уравнений взаимодействующих полей: скалярного $\varphi$ и спинорного $\Theta$ с компонентами $\Theta_{ \pm}=\sqrt{\rho_{ \pm}} \exp \left( \pm 4 i \alpha_{ \pm}\right)$. 
Если $\rho_{ \pm}>0$, то, как показано в работе [7], решение задачи Коши для системы (9)-(11) строится по решению таковой для уравнения (9). Заметим, что лишь в случае, когда функции $\rho_{ \pm}$не обрашаются в нуль, преобразованиями (1) возможно сделать эти функции постоянными всюду и, следовательно, свести (9) к УЛ. В нашем случае функции $\rho_{ \pm}\left(\xi_{ \pm}\right)$являются динамическими переменными (канонически-сопряженными для "конфигурационных" полей $\alpha_{ \pm}$), так что ограничения типа неравенств на них отсутствуют.

Система (9)-(11) обладает широкой группой инвариантности $\mathcal{G}$, которая обобщает группу (1). Действительно, пусть $f_{ \pm}, g_{ \pm}$и $A_{ \pm}$, где $A_{-}^{\prime} A_{+}^{\prime} \neq 0,-$ произвольные дифференцируемые функции. Тогда преобразование

$$
\left(\varphi, \rho_{ \pm}, \alpha_{ \pm}\right) \longrightarrow\left(\widetilde{\varphi}, \widetilde{\rho}_{ \pm}, \widetilde{\alpha}_{ \pm}\right)
$$

вновь дает решение рассматриваемой системы, если

$$
\begin{aligned}
\widetilde{\varphi}\left(\xi_{+}, \xi_{-}\right) & =\varphi\left(A_{+}\left(\xi_{+}\right), A_{-}\left(\xi_{-}\right)\right)+f_{+}\left(\xi_{+}\right)+f_{-}\left(\xi_{-}\right), \\
\widetilde{\rho}_{ \pm}\left(\xi_{ \pm}\right) & =\rho\left(A_{ \pm}\left(\xi_{ \pm}\right)\right) A_{ \pm}^{\prime}\left(\xi_{ \pm}\right) \exp \left(-f_{ \pm}\left(\xi_{ \pm}\right)\right), \\
\widetilde{\alpha}_{ \pm}\left(\xi_{+}, \xi_{-}\right) & =\alpha_{ \pm}\left(A_{+}\left(\xi_{+}\right), A_{-}\left(\xi_{-}\right)\right) \exp \left(f_{ \pm}\left(\xi_{ \pm}\right)\right)+g_{ \pm}\left(\xi_{ \pm}\right) .
\end{aligned}
$$

Преобразования вида $\alpha_{ \pm} \rightarrow \alpha_{ \pm}+g_{ \pm}$в точности соответствуют произволу (4) в выборе светоподобных векторов $n_{ \pm}^{\mu}$, а преобразование $\varphi \rightarrow \varphi+f_{+}+f_{-}-$преобразованию 2-метрики мирового листа. Действительно, используя тот факт, что матрица $K\left(\xi^{0}, \xi^{1}\right) \in S L(2, R)$ связывает меж ду собой матрицы $\widehat{\mathbf{E}}_{ \pm}\left(\xi_{ \pm}\right)$, а также учитывая определения этих вектор-матрищ, для первой квадратичной формы мирового листа мы имеeм

$$
\mathbf{I}=\partial_{+} X^{\mu} \partial_{-} X_{\mu} d \xi_{+} d \xi_{-}=-\frac{p^{2}}{2} e^{-\varphi} d \xi_{+} d \xi_{-} .
$$

Заметим, что в соответствии с (13) преобразования 2-метрики не всегда связаны с переопределением (1) параметров $\xi_{ \pm}$, что вследствие известной вейлевской инвариантности в теории струн является вполне естественным. Для второй квадратичной формы мирового листа находим

$$
\mathbf{I I}=p\left(\rho_{+} d \xi_{+}^{2}-\rho_{-} d \xi_{-}^{2}\right),
$$

так что уравнения (9) и (10) суть не что иное, как уравнения Гаусса и Петерсона-Кодацци, записанные для мирового листа $3 D$-струны [1]. Как уже отмечалось, преобразованиями (1) мы можем добиться выполнения равенств $\rho_{ \pm}=$const и свести (9)-(11) к УЛ, наложив ограничение $\rho_{+} \rho_{-} \neq 0$. То, что такое ограничение является сушественным, следует из принципиальных отличий, которые полученная система имеет по отношению к УЛ. Данные отличия следующие:

1. Имеется вырожденный трансляционно-инвариантный "вакуум" $\varphi \equiv$ const, $\alpha_{ \pm} \equiv$ const, $\rho_{ \pm} \equiv 0$ [7]. Отсутствие такового у УЛ всегда приводило к дополнительным сложностям, в частности при квантовании (см., например, [8]). Заметим, что указанному "вакуумному" решению системы (9)-(11) отвечает прямолинейная струна, движушаяся с постоянной линейной скоростью. 
2. Структура множества сингулярных точек какого-либо решения системы (9)-(11) является, вообще говоря, более сложной. Действительно, с помошью методов, развитых в работе [9], можно показать, что множество $\Re$ точек $\xi^{0}, \xi^{1}$, удовлетворяющих условию (8), содержит в себе линии, которые могут быть времениподобными в $\left(\xi^{0}, \xi^{1}\right)$-плоскости (там, где $\left.\rho_{+} \rho_{-}>0\right)$, пространственноподобными (где $\left.\rho_{+} \rho_{-}<0\right)$, а также замкнутыми, поскольку возможно чередование и тех, и других областей. В случае открытой $3 D$-струны такие типы особенностей функции $\varphi\left(\xi^{0}, \xi^{1}\right)$ полностью соответствуют сингулярностям мирового листа, исследованным в работе [10]. Интересно, что для решений системы (9)-(11) допустимы и иные типы особенностей. Так, например ${ }^{2)}$, если $\rho_{+} \rho_{-} \equiv 0$ на множестве $\Xi=\left[a_{+}, b_{+}\right] \times\left[a_{-}, b_{-}\right]$, где $a_{ \pm}<b_{ \pm}$, то возможно включение $\Xi \subset \Re$. Если мы требуем, чтобы при любом $\xi^{0}$ функции $\varphi\left(\xi^{1}\right), \alpha_{ \pm}\left(\xi^{1}\right)$ имели сингулярности только в точках, то мы должны считать, что для указанных решений киральное поле $K\left(\xi^{0}, \xi^{1}\right)$ определено лиш на подмножестве $\left(\xi^{0}, \xi^{1}\right)$-плоскости, получаемом исключением из нее всех прямоугольников $\left(a_{+}, b_{+}\right) \times\left(a_{-}, b_{-}\right)$. В случае, если хотя бы для одного такого прямоугольника все числа $a_{ \pm}, b_{ \pm}$конечны, такое подмножество не является односвязным. По-видимому, такие решения могут быть использованы для описания мировых листов, которые имеют разрезы (конечные или бесконечные), т.е. для описания распада и слияния струн.

3. Динамическая система (9)-(11) имеет иную по отношению к УЛ гамильтонову структуру. Подробные результаты, касающиеся данного вопроса в общем (сингулярном) случае, получены в работе [11], где для исследования указанной системы применен метод обратной задачи.

С помощью формулы (5) и определения вектор-матрищ $\widehat{\mathbf{E}}_{ \pm}$введенные в начале статьи векторы $\mathbf{n}_{ \pm}$можно представить в виде $n_{ \pm}^{\mu}=(1 / 2) \bar{\Psi}_{ \pm} \Gamma^{\mu} \Psi_{ \pm}$, где

$$
\Psi_{+}= \pm\left(\begin{array}{c}
-t_{12+} \\
t_{11+}
\end{array}\right), \quad \Psi_{-}= \pm\left(\begin{array}{c}
-t_{22-} \\
t_{21-}
\end{array}\right), \quad t_{i j \pm} \equiv\left(T_{ \pm}\right)_{i j}
$$

Как уже отмечалось, преобразования $T_{ \pm} \rightarrow T_{ \pm} B$, где $B \in S L(2, R)$, являются лоренцпреобразованиями пространства $E_{1,2}$, так что объекты $\Psi_{ \pm}-$майорановские спиноры в данном пространстве. Эти спиноры могут быть рассмотрены как дополнительные (по отношению к $X_{\mu}$ ) струнные переменные. Таким образом, предложенная теория описывает либо бозонную струну в произвольной конформно-плоской параметризации, либо некоторую спиновую струну, причем формулу (2) в этом случае необходимо рассматривать как дополнительное условие, фиксируюшее произвол (1). Различные точки какой-либо орбиты группы $\mathcal{G}$ в такой теории будут отвечать различным струнным конфигурациям $\left(X_{\mu}, \Psi_{ \pm}\right)$. Модель в “спиновой” интерпретации была рассмотрена ранее автором в работе [12].

Как было замечено в работе [1], существует альтернативная возможность гамильтонова описания струнной динамики в терминах геометрических переменных, приводяшая к иным физическим результатам. Действительно, в нашем случае орбиты группы $\mathcal{G}$,

\footnotetext{
${ }^{2)}$ Автор благодарен А. К. Погребкову, указавшему ему на сушествование такого типа особенностей.
} 
действуюшей в пространстве сингулярных полей $\varphi\left(\xi^{0}, \xi^{1}\right)$ и $\alpha_{ \pm}\left(\xi^{0}, \xi^{1}\right)$, параметризуют множество струнных конфигураций $X_{\mu}\left(\xi^{0}, \xi^{1}\right)$ взаимно однозначно с точностью до движений и масштабных преобразований пространства $E_{1,2}$. Следовательно, гамильтонова структура, каноническая для действия (12), описывает также и динамику исходных струнных переменных $X_{\mu}$, удовлетворяюших условиям $\left(\partial_{ \pm} X\right)^{2}=0$. Между тем такая структура не является канонической для обычного струнного действия.

В заключение заметим, что случай $4 D$-струны рассматривается аналогично. Отличие состоит в том, что вводимые функции $\varphi$ и $\alpha_{ \pm}$, а также параметризующие калибровочный произвол функции $f_{ \pm}$и $g_{ \pm}$будут комплекснозначными. При этом метрика на мировом листе определяется квадратичной формой

$$
\mathbf{I}=-\frac{p^{2}}{2} e^{-\operatorname{Re} \varphi} d \xi_{+} d \xi_{-} ;
$$

пара же вторых квадратичных форм $\mathbf{I I}_{i}$, где $i=1,2$, компактно записывается в комплексном виде

$$
\mathbf{I I}_{1}+i \mathbf{I I}_{2}=p\left(\left|\rho_{+}\right| e^{i[\beta+\chi]} d \xi_{+}^{2}-\left|\rho_{-}\right| e^{i[\beta-\chi]} d \xi_{-}^{2}\right),
$$

где $\chi \equiv\left(\operatorname{Im} \varphi+\arg \rho_{+}+\arg \rho_{-}\right) / 2$, а число $\beta \in[0,2 \pi)$ произвольно. Как показано в работе [13], с помошью преобразований (13) всегда можно сделать так, что $K\left(\xi^{0}, \xi^{1}\right) \in$ $S U(2)$. Выписывая явные выражения векторов $\partial_{ \pm} x$ через матричные элементы $t_{i j}$ (см. [12]), легко убедиться, что такой выбор соответствует известной калибровке $X^{0}=\xi^{0}$ в теории струн (для выбранных нормировок $X^{0}=p \xi^{0}$ ).

Теория спиновой струны в $4 D$-пространстве-времени, основанная на нестандартном (в указанном смысле) гамильтоновом формализме и приводяшая соответственно к иному спектру, построена в ряде работ автора (см, например, [14]). В этой связи уместно отметить статью [15], где обсуждается возможность описания динамических систем со связями первого рода без фиксации калибровки.

\section{Список литературы}

[1] Б. М. Барбашов, В. В. Нестеренко. Модель релятивистской струны в физике адронов. М.: Энергоатомиздат, 1987.

[2] Б. М. Барбашов, В. В. Нестеренко, А. М. Червяков. ТМФ. 1979. Т. 40. № 1. С. 15.

[3] Г. П. Пронько, А. В. Разумов, Л. Д. Соловьев. ЭЧАЯ. 1983. Т. 14. № 3. С. 558.

[4] С. П. Новиков. ДАН СССР. 1981. Т. 260. № 1. С. 31.

[5] E. Witten. Commun. Math. Phys. 1984. V. 92. P. 455.

[6] Л.А. Тахтаджян, Л. Д. Фаддеев. Гамильтонов подход в теории солитонов. М.: Наука, 1986.

[7] А.К. Погребков, С. В. Талалов. ТМФ. 1987. Т. 70. № 3. С. 342.

[8] E. D'Hoker, R. Jackiw. Phys. Rev. D. 1982. V. 26. P. 3517.

[9] Г. П. Джорджадзе, А. К. Погребков, М. К. Поливанов. ТМФ. 1979. Т. 40. № 2. С. 221.

[10] С. В. Клименко, И. Н. Никитин. ТМФ. 1998. Т. 114. № 3. С. 380.

[11] С. В. Талалов. ТМФ. 1987. Т. 71. № 3. С. 357.

[12] С. В. Талалов. ТМФ. 1989. Т. 79. № 1. С. 41.

[13] С. В. Талалов. ТМФ. 1992. Т. 93. № 3. С. 506.

[14] S. V. Talalov. J. Phys. A. 1999. V. 32. P. 845.

[15] S. I. Muslih, Y. Güler. Nuovo Cimento B. 1998. V. 113. № 3. P. 277.

Поступила в редакцию 17.V.1999 г. 